\title{
Major stimulus dimensions determining detection of simulated breast lesions
}

\author{
H. S. BLOOM, E. L. CRISWELL, and H. S. PENNYPACKER \\ University of Florida, Gainesville, Florida
}

A. C. CATANIA

University of Maryland, College Park, Maryland

and

\author{
C. K. ADAMS \\ Simpson, Kansas
}

\begin{abstract}
Five adult humans palpated silicone breast models in a lump-detection task. The effects on detection of several lump and model characteristics were studied in three phases, using both discrete trial (restricted search procedure) and "free response" (free search procedure) psychophysical methods. Size and depth of fixed steel lumps were varied in Phase 1. Depth and hardness of uniformly sized, fixed lumps were varied in Phase 2. The presence and depth of simulated breast nodularity were also studied in Phases 1 and 2. In Phase 3, all breast models were uniformly nodular and lumps varied along dimensions of size, depth, hardness, and fixation. In Phase 1, lump detection was greatest with maximum lump size and minimum depth within the model. Neither lump hardness nor depth differentially influenced detection of the fixed lumps in Phase 2. When breast lumps were mobile, in Phase 3, size and hardness were major stimulus dimensions determining detection. These results suggest physical parameters for realistic breast models and lumps to be used in training effective breast selfexamination and breast lump detection.
\end{abstract}

Breast cancer is the leading cause of cancer death in women. In 1980 , approximately 36,000 women were expected to die of breast cancer (Cancer Facts and Figures, 1980). In general, the earlier a cancer is detected and treated, the better is the prognosis. The probabilities of both recurrent disease and axillary lymph node involvement increase with increases in primary tumor size (Fisher, Slack, \& Bross, 1969; Haagensen, 1971; Schottenfield, Nash, Robbins, \& Beattie, 1976; Spratt \& Donegan, 1967), and smaller tumors at the time of surgery are correlated with higher 5-year survival rates (Adair, Berg, Joubert, \& Robbins, 1974; Delario, 1959; Fisher et al., 1969; Fisher, Slack, Katrych, \& Wolmark, 1975; Goldenberg, Bailer, Hayes, \& Lowry, 1961; Haagensen, 1971; Say \& Donegan, 1974; Spratt \& Donegan, 1967; Treves \& Hoeleb, 1958).

Although breast self-examination (BSE) has received considerable attention as a means of early detection of breast cancer, empirical data fully describ-

This research was supported by Grant CA-20791 from the Division of Cancer Control of the National Cancer Institute. The authors gratefully acknowledge a helpful earlier reading of the manuscript by Keith White. Requests for reprints should be addressed to H. S. Pennypacker, Center for Ambulatory Studies, 114 Psychology Building, University of Florida, Gainesville, Florida 32611. ing relations between optimum palpation and detection skill and clinical parameters of tumors are lacking. Such information is required if a sound training technology for BSE is to become available. Two studies have been reported, however, in which a partial data base was established. Adams, Hall, Pennypacker, Goldstein, Hench, Madden, Stein, and Catania (1976) determined that detection of lumps simulating cancer in very simple breast models is a task amenable to experimental analysis. When 16 naive observers palpated silicone breast models containing lumps 1.6 to $12.1 \mathrm{~mm}$ in diameter, detectability depended on lump size according to a systematic psychophysical function. Performance improved with practice and showed no appreciable decrement in 3-week follow-up measures. Detection plateaued in 20 to $30 \mathrm{~min}$, suggesting that efficient training can be brief.

In the second report, the simple breast model was also used in a validation-field study (Hall, Stephenson, Adams, Goldstein, Pennypacker, \& Stein, 1980). Before and after a brief experimental training session on the model with simulated lumps, 20 trainees examined six women with a total of 13 known benign breast lesions. The mean lumps detected doubled after model training (from $25 \%$ to $50 \%$ of the 13 lesions), suggesting that variables relevant to effective BSE training were under study. 
In both these studies, the materials simulating breast tissue and lumps were homogeneous silicone gel and steel spheres, respectively. The lumps were fixed at the base of the model, analogous to a latestage cancer affixed to the chest wall. Real breast tissue, however, exhibits some degree of nodularity (tactile heterogeneity), and tumors are not always affixed to the chest wall and can be of variable hardness and mobility. Training for detection of early cancers by palpation may therefore require a model simulating these characteristics. Moreover, an effective BSE training technology presupposes knowledge of the effects of variation of these characteristics on detection. The present experiment determined the effects of depth of lump (Phases 1, 2, and 3), hardness of lump (Phases 2 and 3), and mobility of lump (Phase 3) on detection accuracy.

\section{GENERAL METHOD}

\section{Participants}

Five adult humans were paid for participating in the experiment. P1, P3, and P5 were, respectively, 32-, 21-, and 22-year-old white males, and $P 2$ and P4 were, respectively, 26- and 22-year-old white females. In Adams et al. (1976), no sex- or age-related differences were found. All were experimentally naive and reported neither prior participation in similar studies nor prior regular and systematic BSE. All initially signed a contract that outlined payment contingencies: payment for each session was \$1.50, with an additional 504 for each session if attendance during the week was perfect. Perfect attendance for the entire period of the contract earned a further bonus that raised the payment for each session to \$3.00. Participants signed new payment contracts before each block of $\mathbf{2 0}$ to $\mathbf{3 0}$ sessions and were paid at approximately monthly intervals. Sessions were scheduled three to six times a week (no more than one per day), depending on each participant's availability. Toward the end of the experiment, two sessions per day separated by a minimum of $6 \mathrm{~h}$ were scheduled for P4. Sessions typically lasted $1 \mathrm{~h}$.

\section{Apparatus}

Breast model. The breast model in this study differed significantly from the hemispherical model previously reported. To locate lumps and nodularity (simulated nonhomogeneous breast tissue) at different depths in the model, three separate horizontal model sections were positioned on top of each other. Both clean (i.e., homogeneous interior gel) and nodular sections were used. Lump stimuli in Phase 1 were steel spheres. For Phases 2 and 3 , lumps were cut from a variety of plastics and sponges obtained from the Rex Gauge Co., Inc., Glenview, Illinois. The hardness of these lumps, as determined by indentation resistance, was measured with a Type 00 durometer (Standard Dial Model 1600, Rex Gauge Co.). The higher the durometer reading, the harder the material measured.

Lump configurations were positioned between model sections in Phases 1 and 2 . At Level 1, lumps are positioned only under the bottom section; at Level 2, they are between the bottom and middle section; and at Level 3, they are between the middle and top model section.

In Phase 3, the variable hardness lumps were embedded in, rather than located between, model sections. This allowed these lumps to vary in lateral mobility. All model sections in Phase 3 were nodular.

Technical details regarding model and lump configuration design and construction are noted in Appendix 1.
Setting. All sessions were conducted by E.C. or H.B. in the same room of the Psychology Department. Participant $(P)$ and experimenter (E) sat opposite each other at a small table. Because the models were transparent, a $1.0-\mathrm{m}$ square, vertical black felt screen with armhole slits was secured on the table to eliminate visual cues. The model rested on a $20.8 \times 20.8 \times 6.4 \mathrm{~cm}$ platform covered with foam padding.

\section{Procedure}

In each phase, two search procedures were used: restricted and free. Restricted search was procedurally a single-interval, "yes-no" detection task. On each trial in the restricted search condition, $E$ placed $P$ 's fingers at a specific location on the model. $P$ palpated only that area and responded "yes" or "no," depending on whether or not a lump was detected. Both lump and no-lump trials were scheduled.

In the free-search condition, the detection task was significantly more complex (and more analogous to search behavior during BSE). On each trial, $P$ examined the model once around in a clockwise direction. A timer operated by $E$ signaled the start and end of each trial. When a lump was detected, $P$ held a finger directly on the lump for 2 sec. With the other hand, $P$ pressed a button which produced a short tone.

Before collecting data in the free-search condition, an overall search pattern was trained to minimize acquisition effects as much as possible. Search pattern training is completely described in Appendix 2.

\section{PHASE 1}

\section{Method}

A total of 24 steel lump diameters were used: $.4, .8$ to 1.6 , and 2.0 to 3.2 in .2-mm steps, 3.6 to 7.2 in .4-mm steps, and $12.0 \mathrm{~mm}$. A different series of seven lumps of consecutive size from this overall size range was presented at each level. The largest series was presented at Level 1 and the smallest at Level 3.

Restricted search. Each lump in each series was presented five times at its respective level. Five no-lump trials were presented randomly at each level. This resulted in $\mathbf{4 0}$ trials per level and 120 trials per session. Trials were blocked sequentially by level. The order was 3-2-1 for P1 and P3 and was reversed for P2 and P4 (P5 did not participate in the restricted search portion of Phase 1). Data were collected with the restricted search procedure with each participant until three consecutive sessions had occurred with no more than a .5-mm change in threshold at any level and no major trends in other dependent measures.

Free search. In this procedure, $P$ completed one search of the entire model during each trial. For each trial, the lump configuration contained either four or five lumps randomly distributed (within level) with respect to size and location. The seven lump sizes were presented five times at each level (as in restricted search). These 35 lumps were distributed across eight lump configurations. Consequently, 24 configurations, or trials, were presented during each session (eight trials per level).

The baseline condition in free search was defined by blocking trials sequentially by level. The order of levels was 3-2-1 for P1, $P 3$, and P5 and was reversed for P2 and P4. Stable responding was defined as no more than a $.5-\mathrm{mm}$ change in threshold for three consecutive sessions at all levels and no major trends in other dependent measures. Four other experimental conditions were examined. Table 1 shows the experimental conditions and numbers of sessions for each $\mathrm{P}$ in Phase 1.

\section{Results}

Restricted search. Lump detection threshold was measured in each level of the model. Threshold was calculated by linear interpolation for each session 
Table 1

Experimental Conditions and Number of Sessions in Phases 1-3

\begin{tabular}{|c|c|c|c|c|}
\hline \multirow[b]{2}{*}{ Condition } & \multicolumn{4}{|c|}{$P$} \\
\hline & 1 & 2 & 3 & 5 \\
\hline
\end{tabular}

Phase 1-Restricted Schedule
A. Trials blocked by level*; no nodularity
B. Trials blocked by level*; no nodularity
C. Trials randomized by level; no nodularity
D. As in B; nodularity in Level 1
E. As in B; nodularity in Level 2
$\mathrm{F}$. As in B; nodularity in Level 3

Phase $1-$ Free Schedule

Phase 2-Restricted Schedule

A. Trials blocked by level $*$ proportion of no-lump trials $=18.7 \%$; no nodularity

B. As in A; proportion of no-lump trials $=50 \%$; no nodularity

C. As in A; nodularity in Level 1

D. As in B; nodularity in Level 1

E. As in A; nodularity in Level 2

F. As in B; nodularity in Level 2

G. As in A; nodularity in Level 3

H. As in B; nodularity in Level 3

Phase 2-Free Schedule

1. Trials blocked by level,* 5 lump stimuli; nodularity in Level 1

J. As in I; nodularity in Level 2

K. As in $I$; nodularity in Level 3

L. As in $1 ; 13$ lump stimuli; nodularity in Level 1

M. As in L; nodularity in Level 2

$\mathrm{N}$. As in L; nodularity in Level 3

Phase 3-Restricted Schedule

A. Trials blocked by level* and lump size*; proportion of notump trials $=50 \%$; lump sizes $=3.0,5.0 \mathrm{~mm}$

B. As in A; lump sizes $=5.0,10.0 \mathrm{~mm}$

C. As in $A$; lump sizes $=7.0,10.0 \mathrm{~mm}$

D. As in A; lump sizes $=3.0,5.0,10.0 \mathrm{~mm}$

Phase 3-Free Schedule

E. Trials blocked by level* and lump size*; lump sizes $=5.0,10.0 \mathrm{~mm}$

$\mathrm{F}$. As in $\mathrm{E}$; lump sizes $=3.0,5.0,10.0 \mathrm{~mm}$

$\begin{array}{rrrrr}7 & 4 & 4 & 5 & \\ 29 & 34 & 44 & 30 & 21 \\ 14 & 21 & 1 & 10 & 11 \\ 1 & 2 & 1 & 2 & 1 \\ 1 & 2 & 1 & 3 & 1 \\ 1 & 2 & 1 & 1 & 1\end{array}$

*See text for order.

from the psychophysical functions relating the percent of lumps detected to lump size. Means and standard deviations of the threshold measure from the last four sessions of this procedure are shown in $\mathrm{Ta}$ ble 2. For all participants, threshold and variability were highest at Level 1 and lowest at Level 3.

Table 2

Restricted Search Procedure: Threshold Means and Standard Deviations (in Millimeters) Across the Last Four Sessions of Phase 1

\begin{tabular}{|c|c|c|c|c|c|c|c|c|}
\hline \multirow[b]{3}{*}{ Level } & \multicolumn{8}{|c|}{ Participant } \\
\hline & \multicolumn{2}{|c|}{ P1 } & \multicolumn{2}{|c|}{ P2 } & \multicolumn{2}{|c|}{ P3 } & \multicolumn{2}{|c|}{ P4 } \\
\hline & Mean & $\mathrm{SD}$ & Mean & SD & Mean & SD & Mean & SD \\
\hline 1 & .77 & .12 & .77 & .13 & .99 & .12 & 1.00 & .18 \\
\hline 2 & .62 & .03 & .61 & .02 & .64 & .07 & .71 & .10 \\
\hline 3 & .56 & .02 & .59 & .02 & .55 & .06 & .52 & .05 \\
\hline
\end{tabular}

Note-- "Level" = level of lump placement.
Measures of performance accuracy from the same sessions are shown in Table 3. The first number in each entry of the table is the total number of "yes" detection responses, and the second number is the percentage that were hits (lump present). Table 3 suggests that the level at which lumps were presented did not differentially affect detection accuracy in the discrete trial procedure.

Free search. Threshold means and standard deviations for P1 and P5 from the last four sessions of baseline are shown in Figure 1. The inverse relation between threshold and level that obtained in the discrete trial procedure (see Table 2) also did so in this "free-response" situation. The threshold ranges across participants at Levels 1, 2, and 3, respectively, were .95 to $1.5 \mathrm{~mm}, .75$ to $1.4 \mathrm{~mm}$, and .6 to $1.1 \mathrm{~mm}$.

Table 4 shows measures of performance accuracy during free search from the same four baseline sessions. These data show that only in the cases of $\mathrm{P} 2$ and P5 did detection accuracy drop below $100 \%$. In 
Table 3

Restricted Search Procedure: Total Number (N) of "Yes" Detection Responses and Percent (\%) of Those That Were Hits Across the Last Four Sessions of Phase 1

\begin{tabular}{|c|c|c|c|c|c|c|}
\hline \multirow{3}{*}{$\begin{array}{c}\text { Partici- } \\
\text { pant }\end{array}$} & \multicolumn{6}{|c|}{ Lump Placement } \\
\hline & \multicolumn{2}{|c|}{ Level 1} & \multicolumn{2}{|c|}{ Level 2} & \multicolumn{2}{|c|}{ Level 3} \\
\hline & $\mathbf{N}$ & $\%$ & $\mathrm{~N}$ & $\%$ & $\mathbf{N}$ & $\%$ \\
\hline $\begin{array}{l}\text { P1 } \\
\text { P2 } \\
\text { P3 } \\
\text { P4 }\end{array}$ & $\begin{array}{l}112 \\
106 \\
109 \\
108\end{array}$ & $\begin{array}{r}100 \\
98 \\
100 \\
97\end{array}$ & $\begin{array}{l}123 \\
123 \\
116 \\
111\end{array}$ & $\begin{array}{r}100 \\
97 \\
98 \\
99\end{array}$ & $\begin{array}{l}121 \\
123 \\
123 \\
132\end{array}$ & $\begin{array}{r}100 \\
98 \\
98 \\
96\end{array}$ \\
\hline
\end{tabular}

each case, the greatest drop occurred at Level $1(78 \%$ and $84 \%$, respectively). Accuracy was $89 \%$ or above at Levels 2 and 3.

Mean trial duration varied little within participant and was not consistently related to the level of lump placement. For P1-P5, duration ranges were (in seconds) respectively, 85-92, 65-70, 96-100, 135-149, and 59-72.

The change from presenting sequential blocks of trials across levels (baseline) to randomizing levels across trials had no major lasting effects on performance. The change initially induced variability in the dependent measures for all but P3, but the measures soon stabilized around baseline values. (Since no dependent measure showed significant change for P3, only one session was run.)

The use of steel lumps in Phase 1 permitted partial replication and comparisons with earlier studies. The introduction of nodular sections did not disrupt detection. Such a result is probably due to the highly discriminable nature of the steel lumps and the observers' extended history with the task when these conditions were introduced. This finding suggested the continued use of nodular sections to enhance the fidelity of the breast model.

The fidelity of the materials (vis-à-vis human breast tissue) in Phase 1 was judged inadequate for deployment in any mass BSE training effort. Phase 2 was designed to determine the effects on detection of introducing lumps whose firmnesses more closely approximated those of actual cysts and tumors.

\section{PHASE 2}

\section{Method}

The primary change from Phase 1 to Phase 2 was the nature of the lumps. The variable hardness lumps in Phase 2 were hand cut from larger samples of the materials and were approximately $3.0 \mathrm{~mm}$ on a side. Preliminary testing with naive observers showed that any larger sizes were almost invariably detected; the $3.0-\mathrm{mm}$ size was the smallest that could be cut from the foams and sponges with any precision. The lumps, glued to a silicone rubber sheet $.3 \mathrm{~mm}$ thick, were presented at three levels, as in Phase 1 .

Thirteen different lump hardnesses were used. The durometer readings were: $6.0,7.5,9.0,11.0,14.0,15.0,20.0,24.0,35.0$, $38.0,47.0,51.0$, and 44.0.' The silicone materials used to simulate breast nodularity had a durometer reading of 11.0.

Restricted search. Each of the 13 lumps was presented five times in random order at each level. No-lump trials were scheduled $50 \%$ of the time for $\mathbf{P 2}$ in all sessions. These parameters resulted in 130 trials per level. P3 and P4 also received no-lump trials $18.7 \%$ of the time for those sessions indicated in Table 1, during which there were 80 trials per level. Table 1 shows the experimental conditions during this part of Phase 2 . Nodularity was either absent or present in one model section in each condition. Trials were always blocked sequentially according to level (3-2-1 for P1, P3, and P5; $1-2-3$ for P3 and P4).

Free search. For P3 and P4, the same 13 lump hardnesses were distributed across three lump configurations. Each configuration contained four or five lumps of differing hardness. Hardness was randomized within configuration. Lumps were located in the middle third of four or five equally spaced radii (one lump per radius). Each configuration was presented five times per level, and this

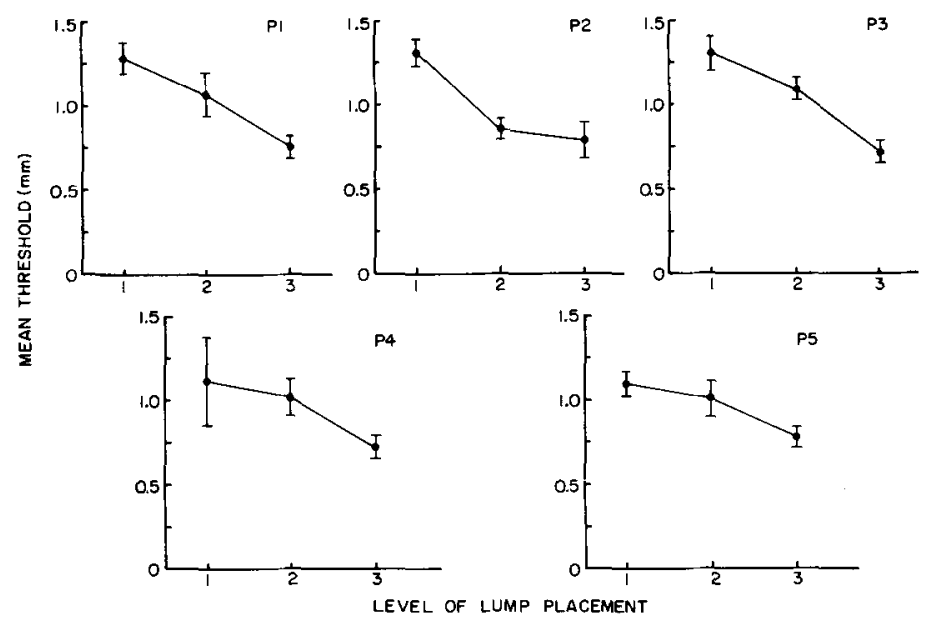

Figure 1. Mean and standard deviation (vertical lines) of threshold in millimeters for each participant at each level in the model. The data are based on the last four baseline sessions of the Phase 1 free-search procedure. 
Table 4

Free Search Procedure: Total Number (N) of "Yes" Detection Responses and Percent (\%) of Those That Were Hits Across the Last Four Sessions of Phase 1

\begin{tabular}{|c|c|c|c|c|c|c|}
\hline \multirow{3}{*}{$\begin{array}{c}\text { Partici- } \\
\text { pant }\end{array}$} & \multicolumn{6}{|c|}{ Lump Placement } \\
\hline & \multicolumn{2}{|c|}{ Level 1} & \multicolumn{2}{|c|}{ Level 2} & \multicolumn{2}{|c|}{ Level 3} \\
\hline & $\mathbf{N}$ & $\%$ & $\mathrm{~N}$ & $\%$ & $\mathrm{~N}$ & $\%$ \\
\hline P1 & 69 & 100 & 87 & 100 & 90 & 100 \\
\hline P2 & 102 & 78 & 88 & 89 & 113 & 89 \\
\hline P3 & 70 & 100 & 71 & 100 & 104 & 100 \\
\hline P4 & 67 & 100 & 74 & 100 & 90 & 100 \\
\hline P5 & 96 & 84 & 95 & 96 & 102 & 95 \\
\hline
\end{tabular}

resulted in $\mathbf{4 5}$ free search trials per session. The configurations were rotated randomly with each presentation.

For P2, only one configuration containing five lumps was presented. The five hardness readings were 11.0, 15.0, 35.0, 51.0, and 55.0. This configuration was presented five times at each level and rotated randomly with each presentation. Three nodularity conditions were run (nodularity presented in each level). Table 1 lists the sessions per condition for P2 to P4.

\section{Results}

Restricted search. The primary finding in this part of Phase 2 was that neither nodularity nor the level at which relatively fixed $3.0-\mathrm{mm}$ lumps of variable hardness were presented had any significant or systematic effect on detection accuracy. Since the hardness of the nodularity mixture was close to or matched the hardness of some lumps, mobility was apparently the relevant stimulus dimension supporting the high accuracy shown in Table 5.

Hit and false-alarm rates are shown in Table 5. The undetected lumps in those sessions showed no orderly relation to the hardness dimension.

Free search. The free-search results essentially replicate those of restricted search in this phase. Neither nodularity nor level exerted strong differential control over detection responding. The data reported are from the final session in each nodularity condition.

Table 6 shows the percent of lumps detected and the corresponding number of false alarms. (The present method of data collection did not allow measurement of correct rejection responses in free search. Consequently, false alarms are reported as raw numbers, not percentages.) The undetected lumps again showed no systematic relation to hardness, and misses can be attributed largely to occasional deficiencies or gaps in the overall search pattern.

Ranges of mean trial duration (in seconds) for P2-P4 were, respectively, 37-71, 95-107, and 85-106. Although P2 had significantly briefer durations, this was not a function of fewer trials per session. P2's trial durations were typically briefer in each phase of the study.

To summarize the results of Phase 2, lump hardness did not influence detectability, and the psycho-

Table 5

Restricted Search Procedure: Percentages of Lumps Detected (\%) and False Alarms (FA) as a Function of Lump and Nodularity Placement

\begin{tabular}{|c|c|c|c|c|c|c|c|c|c|c|c|c|c|c|c|c|c|c|}
\hline \multirow{4}{*}{$\begin{array}{l}\text { Nodularity } \\
\text { Placement }\end{array}$} & \multicolumn{18}{|c|}{ Lump Placement } \\
\hline & \multicolumn{6}{|c|}{ Level 1} & \multicolumn{6}{|c|}{ Level 2} & \multicolumn{6}{|c|}{ Level 3} \\
\hline & \multicolumn{2}{|c|}{ P2 } & \multicolumn{2}{|c|}{ P3 } & \multicolumn{2}{|c|}{$\mathrm{P} 4$} & \multicolumn{2}{|c|}{$\mathrm{P} 2$} & \multicolumn{2}{|c|}{ P3 } & \multicolumn{2}{|c|}{ P4 } & \multicolumn{2}{|c|}{ P2 } & \multicolumn{2}{|c|}{ P3 } & \multicolumn{2}{|c|}{ P4 } \\
\hline & $\%$ & $\mathrm{FA}$ & $\%$ & FA & $\%$ & FA & $\%$ & FA & $\%$ & FA & $\%$ & FA & $\%$ & FA & $\%$ & FA & $\%$ & FA \\
\hline None & 100 & 4 & 89 & $13 *$ & 96 & $0^{*}$ & 100 & 1 & 100 & 20 & 100 & 6 & 100 & 1 & 100 & 2 & 100 & 0 \\
\hline Level 1 & 100 & 12 & 100 & 6* & 100 & 0 & 100 & 9 & 98 & 6 & 100 & 0 & 100 & $i$ & 100 & 13 & 100 & 0 \\
\hline Level 2 & 95 & 10 & 100 & 6 & 100 & 0 & 100 & 12 & 100 & 7 & 100 & 1 & 100 & 4 & 100 & 0 & 100 & 1 \\
\hline Level 3 & 98 & 0 & 100 & 1 & 100 & 0 & 93 & 4 & 100 & 4 & 100 & 0 & 98 & 16 & 100 & 3 & 100 & 0 \\
\hline
\end{tabular}

Note-The data are from the last session of each nodularity condition of Phase 2.

*Based on $18.7 \%$ no-lump trials; all other entries based on $50 \%$ no-lump trials.

Table 6

Free Search Procedures: Percentages of Lumps Detected (\%) and Numbers of False Alarms (FA) as a Function of Lump and Nodularity Placement

\begin{tabular}{|c|c|c|c|c|c|c|c|c|c|c|c|c|c|c|c|c|c|c|}
\hline \multirow{4}{*}{$\begin{array}{l}\text { Nodularity } \\
\text { Placement }\end{array}$} & \multicolumn{18}{|c|}{ Lump Placement } \\
\hline & \multicolumn{6}{|c|}{ Level 1} & \multicolumn{6}{|c|}{ Level 2} & \multicolumn{6}{|c|}{ Level 3} \\
\hline & \multicolumn{2}{|c|}{ P2 } & \multicolumn{2}{|c|}{ P3 } & \multicolumn{2}{|c|}{ P4 } & \multicolumn{2}{|c|}{ P2 } & \multicolumn{2}{|c|}{$\mathbf{P 3}$} & \multicolumn{2}{|c|}{ P4 } & \multicolumn{2}{|c|}{ P2 } & \multicolumn{2}{|c|}{ P3 } & \multicolumn{2}{|c|}{ P4 } \\
\hline & $\%$ & FA & $\%$ & FA & $\%$ & FA & $\%$ & FA & $\%$ & FA & $\%$ & FA & $\%$ & FA & $\%$ & FA & $\%$ & FA \\
\hline Level 1 & 76 & 6 & 95 & 0 & 95 & 1 & 92 & 1 & 98 & 3 & 98 & 1 & 92 & 1 & 97 & 4 & 100 & 1 \\
\hline Level 2 & 76 & 1 & 91 & 0 & 91 & 3 & 68 & 1 & 92 & 0 & 98 & 1 & 84 & 2 & 100 & 1 & 100 & 0 \\
\hline Level 3 & 88 & 3 & 92 & 2 & 86 & 4 & 76 & 1 & 97 & 0 & 95 & 1 & 92 & 0 & 98 & 0 & 95 & 0 \\
\hline
\end{tabular}

Note-The entries refer to the last session of each nodularity condition of Phase 2. 
physical functions were essentially flat with respect to that stimulus dimension. The generality of this finding is, however, limited, given that these participants were highly practiced and the lumps were fixed in the horizontal dimension. The physical dynamics of the real lump detection task involve finger-hand movement, as well as the movement of tissue in response. Masses firmly affixed in the breast are positively correlated with a late-stage clinical status. Because the necessary and sufficient stimulus conditions for "early"' detection of breast masses are the central concern, Phase 3 was conducted. Size, hardness, and depth of lumps were varied. These lumps were embedded in nodular model sections and were mobile under palpation.

\section{PHASE 3}

\section{Method}

In Phase 3, the model-lump arrangement more closely matched the physical properties of the natural situation for the majority of breast lumps. The number of model sections was reduced from three to two to approximate more accurately the distribution of breast tissue over the chest wall in the supine position. Both sections were always nodular.

In Phases 1 and 2, lumps were located between or below the model sections and were fixed in the horizontal dimension. In Phase 3, lumps were not only of variable hardness but were embedded in the sections, resulting in horizontal as well as vertical mobility. Lump diameter was $3,5,7$, or $10 \mathrm{~mm}$. The radius of mobility was $1 \mathrm{~cm}$ for $3-$ and $5-\mathrm{mm}$ lumps, and $.5 \mathrm{~cm}$ for $7-$ and 10-mm lumps.

Five of the 13 lump materials of Phase 2 were used. Durometer readings were: $6.0,14.0,24.0,38.0$, and 51.0 . A section containing lumps had one of each hardness, with size held constant. Lumps were positioned in the middle third of five equally spaced radii. The order of lump hardness within a section was random, and sections were randomly rotated between trials to minimize de- velopment of position bias. Consulting surgeons from the local medical center palpated these models and judged the nodularity and lump characteristics to be very lifelike.

Restricted search. Each lump was presented five times per level, and no-lump trials were always scheduled $50 \%$ of the time. Table 1 shows the experimental conditions for the participants in this part of Phase 3. Conditions were defined by the lump sizes presented. Within each size category, all five hardnesses were presented.

Trials were blocked sequentially, both according to level and lump size. Level order was 1-2 for P2 and P4; it was reversed for P3. Size order was large-to-small for P2 and P3 and was reversed for P4. When three lump sizes were presented, the total number of trials per level was 150 .

Free search. The numbers of sessions for each participant in the two free-search conditions appear in Table 1. On a given trial, lumps were present in only one level. Five lumps were presented in each trial, one of each hardness, with size held constant. Trials were blocked sequentially by level and lump size, with the same order reported in Restricted Search above. In the three-size condition, there were 15 free-search trials per level and 30 per session.

Follow-up data were obtained 6 months after the last session for P3 and P4. P2 had relocated shortly after her last session and was unavailable for follow-up testing. The three-size $(3,5,10 \mathrm{~mm})$ condition was in effect during the follow-up sessions, and trials were blocked as indicated in the preceding paragraph.

\section{Results}

Restricted search. We report only the data from the three-size $(3,5$, and $10 \mathrm{~mm})$ condition. For $P 2$, these data are based on one session, and for P3 and P4 they are based on the last four sessions of this condition.

Hit and false-alarm data appear in Figure 2. This shows the percent of lumps detected calculated on the basis of total lump trials. The percent of false alarms is likewise calculated on the basis of total no-lump trials. Overall, more lumps were detected at Level 2 and more false alarms reported at Level 1.

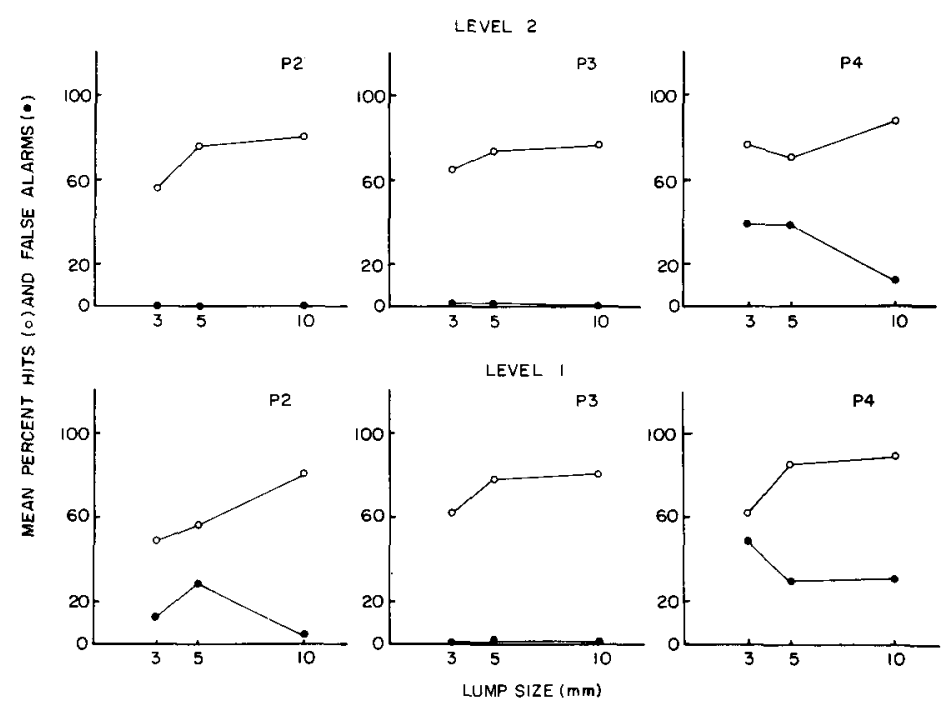

Figure 2. Mean percent lumps detected (open circles) and false alarms (closed circles) for each participant as a function of lump size and lump placement. The data are based on the single session for $P 2$ and the last four sessions for P3 and P4 during the Phase 3 restricted search procedure. 


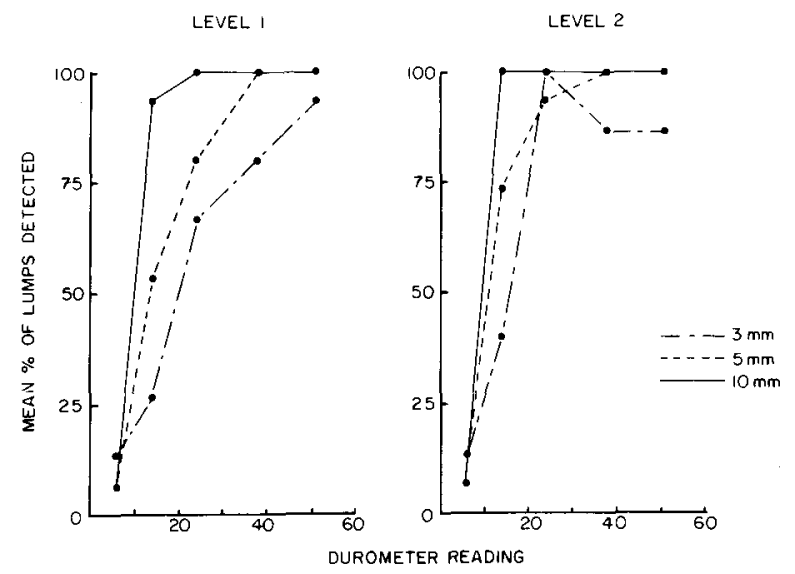

Figure 3. Mean percent of lumps detected at both levels in the model as a function of durometer reading (lump hardness), with lump size as the parameter. Means are based on the data from the last session of Phase 3 restricted search procedure (P2-P4).

The mean psychophysical functions for restricted search are shown in Figure 3. These data are based on the last session in the three-size condition for P2 to P4 and show that detection of mobile lumps generally varies as a function of lump hardness and size.

Free search. The data reported represent both sessions for P2, the last four three-size sessions for P3, and the four two-size sessions for P4. Comparisons of the percent of lumps detected with the number of false alarms may be seen in Table 7 . Percent detected shows a positive relation to lump size, but false alarms and lump size are inconsistently related.

Mean trial duration (in seconds) during these sessions ranged from 36 to 55,150 to 175 , and 122 to 134 for P2, P3, and P4, respectively. Duration was related to neither level nor lump size in a consistent fashion.

Detection data from the last session (prior to followup) for P2 to P4 were averaged to produce the mean psychophysical functions in Figure 4. These plots of free search performance also show that mobile lumps

Table 7

Free Search Procedure: Percentages of Lumps Detected (\%) and Numbers of False Alarms (FA) as a Function of Lump Size (in Millimeters) and Placement

\begin{tabular}{|c|c|c|c|c|c|c|c|c|c|c|c|c|}
\hline \multirow{4}{*}{$\begin{array}{c}\text { Lump } \\
\text { Size }\end{array}$} & \multicolumn{12}{|c|}{ Lump Placement } \\
\hline & \multicolumn{6}{|c|}{ Level 1} & \multicolumn{6}{|c|}{ Level 2} \\
\hline & \multicolumn{2}{|c|}{ P2 } & \multicolumn{2}{|c|}{ P3 } & \multicolumn{2}{|c|}{ P4 } & \multicolumn{2}{|c|}{ P2 } & \multicolumn{2}{|c|}{ P3 } & \multicolumn{2}{|c|}{ P4 } \\
\hline & $\%$ & FA & $\%$ & FA & $\%$ & FA & $\%$ & FA & $\%$ & FA & $\%$ & FA \\
\hline 3.0 & 2 & 8 & 0 & 0 & & & 4 & 17 & 12 & 0 & & \\
\hline 5.0 & 6 & 14 & 40 & 3 & 42 & 2 & 8 & 13 & 49 & 0 & 53 & 18 \\
\hline 10.0 & 40 & 12 & 71 & 0 & 65 & 5 & 34 & 13 & 72 & 0 & 75 & 1 \\
\hline
\end{tabular}

Note-P4 was not presented with $30-\mathrm{mm}$ lumps. See text for sessions represented.

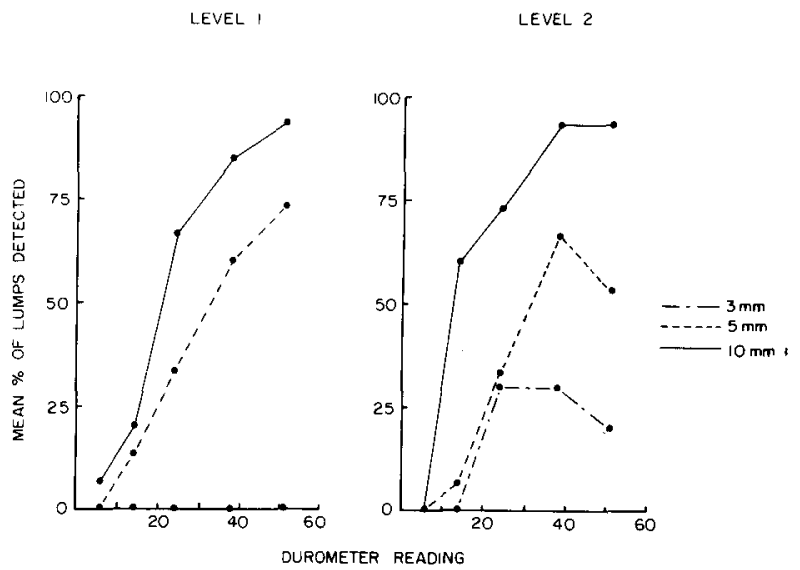

Figure 4. Mean percent of lumps detected at both levels in the model as a function of durometer reading (lump hardness) with lump size as the parameter. Means are based on the data from the last session of the Phase 3 free-search procedure (P2-P4).

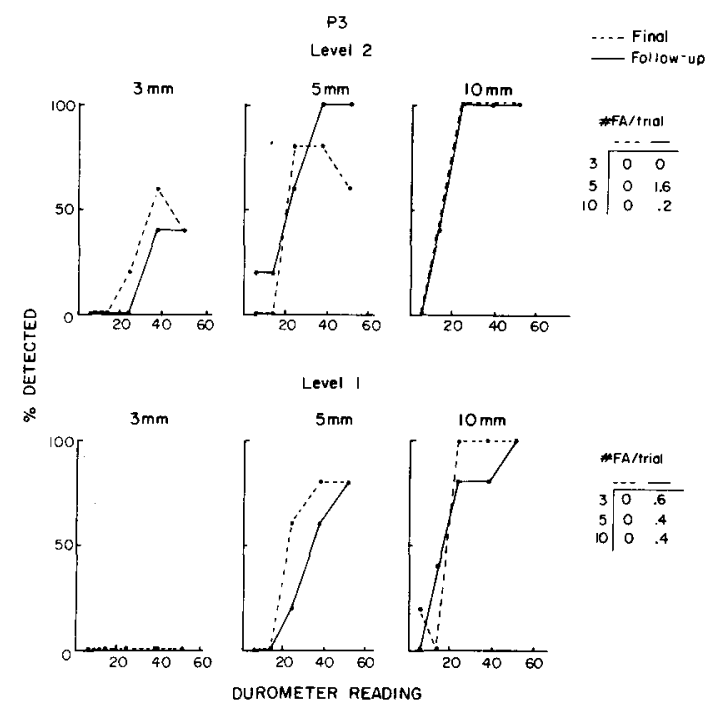

Figure 5. Percent of lumps detected in both levels of the model as a function of durometer reading (lump hardness) with lump size as the parameter. The broken line represents P3's final performance, and the solid line performance after a 6-month no-practice interval. Insets to the right indicate number of false alarms per trial in each lump-size context in both sessions.

are more frequently detected as size and hardness increases.

In Figure 5, the final performance (broken line) and follow-up performance (solid line) of P3 reveal no serious skill loss after a 6-month, no-practice interval. In fact, detection improved when 5-mm lumps were at Level 2. The false-alarm data (inset), however, indicated that this aspect of the discrimination had deteriorated slightly, especially when 5-mm lumps were at Level 2.

This comparison between final and follow-up performance is seen in Figure 6 for P4. Since she did not 


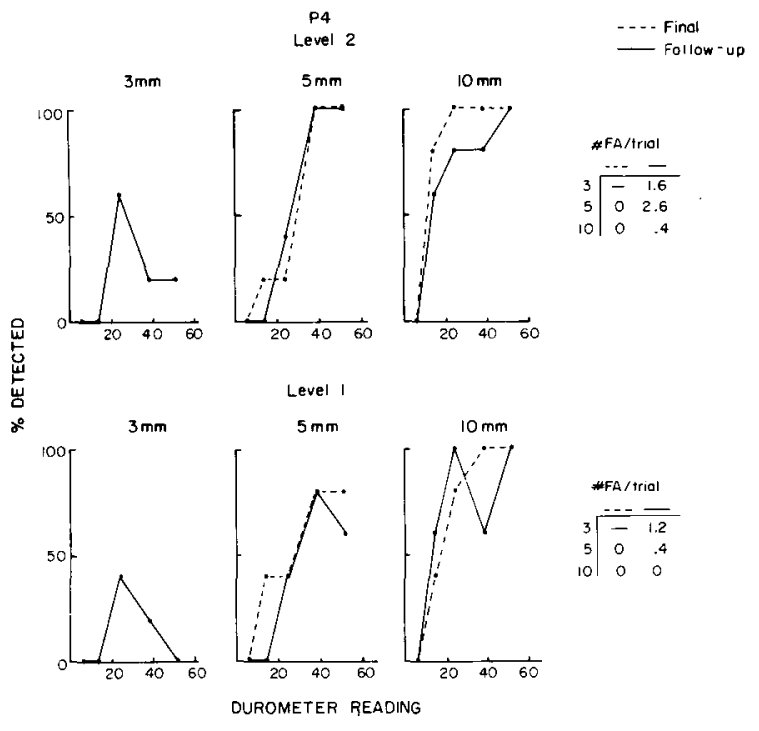

Figure 6. Percent of lumps detected in both levels of the model as a function of durometer reading (lump hardness) with lump size as the parameter. The broken line represents P4's final performance, and the solid line, performance after a 6-month no-practice interval. Insets to the right indicate number of false alarms per trial in each lump-size context in both sessions.

receive the 3-mm size earlier, the 3-mm data in Figure 6 do not describe retention per se. The 5- and 10-mm data essentially replicate the finding with P3 of no serious loss in detection skill after the no-practice interval. Observations during follow-up indicated that deviations from monotonically increasing psychophysical functions depended on the thoroughness of the overall search pattern. Deviations in the functions correspond to areas missed, and consequently lumps missed, by P3 during her search. The general trend of increased false alarms was also reproduced with P4.

While the sessions for each participant in Phase 3 were limited, this methodological concern is tempered by the robust retention effects.

\section{GENERAL DISCUSSION}

The data of this report constitute a major link in a thematically related series of empirical findings. The cumulation of these findings will be the scientific base of a behavioral technology ultimately aimed at reducing the mortality and morbidity consequent to breast cancer.

The experimental question of Phase 1 concerned the effects on detection of the vertical position of the lump. In earlier studies, lumps were located in the lowest position, the simplest design from a manufacturing perspective. In the absence of differential effects on detection, pragmatics would define this design as preferable. Phase 1 data, however, show that differential effects do exist; the effect is small, but the experimental histories of these observers militates against assuming a similar magnitude of effect with naive trainees.

The analysis of lump hardness began with Phase 2. All lumps (varying across 13 grades of hardness) were of the 3-mm size and fixed. These lumps were consistently detected. While exhibiting no differential effect of lump hardness, these data may be summarized alternatively in a statement with encouraging clinical implications. Relatively fixed $3-\mathrm{mm}$ masses were reliably detected with low false-alarm rates, regardless of mass hardness. Malignant tumors tend to be relatively fixed compared with benign tumors (Haagensen, 1971). Haagensen estimated that the average tumor size at the time of surgery is between 3 and $5 \mathrm{~cm}$. The reduction in disease recurrence probability and a less mutilating set of treatment alternatives following diagnosis of a cancer measured in millimeters vs. centimeters are potent components of the general educational theme that must accompany the hands-on training of the proposed technology. Assuming reproducibility, the Phase 2 data cannot therefore be construed as a negative result.

A primary objective of the training technology is delivering to women training in the skill required to detect small breast tumors, be they malignant or benign. Although $80 \%$ of breast tumors are benign, differential diagnosis is decidedly not an objective of this technology, and this activity must obviously be left to members of the medical community. Training detection of all breast tumors (hard and soft) has been emphasized because women with benign cystic disease (softer masses) are estimated to be at a fourfold greater risk of breast cancer (harder masses) than noncystic women (The Breast Cancer Digest, 1979).

Comparing Phase 2 data with the 3-mm data of Phase 3 strongly suggests that mobile lumps are more difficult to detect than fixed lumps. When lumps are mobile, size and hardness emerge as major stimulus determinants of detectability, especially in a context in which one-third of the total model volume was simulated nodularity.

On the basis of the aggregate findings of the present study, a number of quantitative statements can be made regarding the optimal physical parameters of a lifelike breast model necessary for an effective lump detection training technology. Fixed lumps may be as small as $3 \mathrm{~mm}$ in size. Mobile lumps may range from 5 to $10 \mathrm{~mm}$ in size; a lower limit on hardness for mobile lumps is in the range of a 20 to 30 durometer reading. The vertical location of lumps should vary to achieve maximum fidelity.

The maximum amount of nodularity that may be incorporated was not tested here. In earlier, unpublished data, however, we observed a maximum shift in detection threshold only from 1.0 to $1.4 \mathrm{~mm}$ when nodularity was increased from $33 \%$ to $45 \%$, sug- 
gesting that the percentage can effectively be increased well beyond $33 \%$. To enhance positive transfer and maintenance of the new skill, a trainee might best be taught on a model that approximates the characteristics of her own tissue, particularly with respect to firmness and nodularity.

The two search procedures may also be incorporated into the training procedure. Specifically, restricted search more directly assesses detection sensitivity, unhampered by deficits in the overall search pattern. This procedure may be used to initially establish criterion level detection skill, which will be determined individually for each trainee and incorporated into the remainder of her training.

Before any mass dissemination effort is undertaken, it will be necessary to develop training procedures which optimize acquisition of the detection skill. Furthermore, procedures must be devised to insure the transfer and maintenance of the newly acquired skill, or its value in reducing morbidity and mortality from breast cancer will be negligible. Research addressing both of these issues is in progress.

\section{REFERENCES}

Adair, F., Berg, J., Joubert, L., \& Robbins, G. Long-term follow-up of breast cancer patients. Cancer, 1974, 33, 1145-1150.

adams, C. K., Hall, D. C., Pennypacker, H. S., Goldstein, M. K., Hench, L. L., Madden, M. C., Stein, G. H., \& Catania, A. C. Lump detection in simulated human breasts. Perception \& Psychophysics, 1976, 20, 163-167.

The breast cancer digest, Washington, D. C: U.S. Department of Health, Education, \& Welfare, Public Health Service, National Institutes of Health, National Cancer Institute, 1979.

Delakio, A. Breast cancer: Factors modifying prognosis. New York: Macmillan, 1959.

Fisher, B., Slack, N. H., \& Bross, I. Cancer of the breast: Size of neoplasm \& prognosis. Cancer, 1969, 24, 1971-1980.

Fisher, B., Slack, N. H., Katrych, D., \& Wolmark, N. Ten-year follow-up results of patients with carcinoma of the breast in a cooperative clinical trial evaluating surgical adjuvant chemotherapy. Surgical Gynecology \& Obstetrics, 1975, 140, 528-534.

Goldenburg, I. S., Bailer, J. C., Hayes, M. A., \& Lowry, R. Female breast cancer: A reevaluation. Annals of Surgery, 1961, 154, 397-404.

Hangensen, C. Diseases of the breast. Philadelphia: Saunders, 1971.

Hall, D. C., Stephenson, H. S., Adams, C. K., Goldstein, M. K., Pennypacker, H. S., \& Stein, G. H. Improved detection of human breast lesions following experimental training. Cancer, 1980, 46, 408-414.

Madden, M. C., Hench, L. L., Hall, D. C., Adams, C. K., Goldstein, M. K., Pennypacker, H. S., \& Stein, G. H. Development of a model human breast with tumors for use in teaching breast examination. Journal of Bioengineering, 1978, $2,427-435$.

SAY, C. C., \& Donegan, W. L. Invasive carcinoma of the breast: Prognostic significance of tumor size and involved axillary lymph nodes. Cancer, 1974, 34, 468-471.

Schottenfield, D., Nash, A. G., Robbins, G. F., \& Beattie, F. J., JR. Ten-year results of the treatment of primary operable breast carcinoma: A summary of 304 patients evaluated by the TNM system. Cancer, 1976, 38, 1001-1007.

Spratt, J., \& Donegan, W. Cancer of the breast. Philadelphia: Saunders, 1967.
Theves, N., \& Hoeleb, A. I. A report of 549 cases of breast cancer in women 35 years of age or younger. Surgical Gynecology \& Obstetrics, 1958, 107, 271-283.

\section{NOTES}

1. Durometer readings were taken on six excised breast masses; four were malignant (three infiltrating ductal carcinomas and one stromal sarcoma) and two were benign tumors (fibroadenomas). Durometer readings on the malignant tumors ranged from 58 to 73. The fibroadenoma ratings were 38 and 42 . Further data collection on actual tumor hardness is in progress.

2. The term "early" is technically a misnomer with respect to the entire life cycle of the tumor. It is known, from the study of cell kinetics, that a palpable tumor has undergone many reproductive passages and that these events may have occurred over the course of years.

\section{APPENDIX 1}

\section{Model}

All model sections were identical in size and shape (diameter $14.5 \mathrm{~cm}$, height $1.7 \mathrm{~cm}$ ) and had a volume of $140 \mathrm{ml}$. A simulated nipple, $1.5 \mathrm{~cm}$ in diameter and $3.0 \mathrm{~mm}$ thick, was located on the center top of the uppermost section. The basic components of each section were a thin surrounding skin of silicone rubber, an interior of silicone gel, and a back of silicone rubber. See Madden, Hench, Hall, Adams, Goldstein, Pennypacker, and Stein (1978) for a complete description of the model construction process; all details are the same for the present model except size and shape of the mold and material used for making model skins. Skins were made with a silicone elastomer, MDX4-4210, manufactured by the Medical Products Division, Dow Corning Corp., Midland, Michigan. This elastomer was mixed in a ratio of 10:1 with its curing agent before application to the breast model mold. Physical properties of the model skins made with the Dow product are the same as those previously obtained with a General Electric silicone elastomer product.

Although nodules in the nodular sections were made with the same silicone gel formula as the section interior, the curing agent was increased from $6.7 \%$ to $11.3 \%$. This mixture was made in $150-\mathrm{g}$ batches and, after curing, was cut and stirred so that the result looked something like very dry, colorless scrambled eggs. The nodular section differed

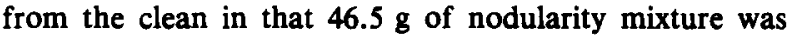
added, with total section volume held constant at $140 \mathrm{ml}$.

\section{Lumps}

Lump configurations in Phase 1 were constructed by encasing the steel spheres between two thin (.3-mm-thick) sheets of the silicone rubber material used for model skins. These sheets adhered to each other when pressed firmly together. In Phase 2 , the variable hardness lumps were affixed with a small dot of silicone glue to a single silicone rubber sheet.

\section{Hardness Measures}

The type 00 durometer is an instrument designed for measuring hardness in a range of soft felt and textile windings and soft animal and vegetable tissue. As an indication of its sensitivity, a piece of felt $.5 \mathrm{~mm}$ thick placed on a Formica top has a durometer 00 reading of 65 (reading for 
Formica = 100); the same piece folded once (1-mm thickness) has a reading of 52 , folded twice (2-mm thickness), a reading of 40 , and folded three times (4-mm thickness), one of 27.

\section{APPENDIX 2}

\section{Search Pattern Tmining}

Initially, the experimenter (E) described and modeled the desired palpation topography: With the first three fingers pressed firmly together at the tips, small circular motions are made with the pads (print area) of the fingertips pressing on the model. $E$ then verbally described the overall pattern of search while drawing it for the participant $(P)$ on a blank sheet of paper. Starting at the three o'clock position on the edge of the model, $P$ was instructed to palpate on an imaginary line to the nipple, back out to the edge on a slightly offset line from the first, along the edge approximately $4 \mathrm{~cm}$, then back to the nipple, continuing in this fashion until reaching the starting point.
E demonstrated the maximum amount of pressure to exert during search by pressing P's fingers down on the model until they stopped against the foam padding supporting the model. This pressure was determined to be within the range of comfort for experimenters during selfexamination. The appropriate measures were obtained with an LX $1601 \mathrm{G}$ hybrid integrated-circuit gauge pressure transducer supplied by National Semiconductor and calibrated by $\mathbf{R}$. G. Thomas, engineering consultant.

Three practice trials followed these demonstrations. $\mathbf{P}$ was instructed to use 1 to $2 \mathrm{~min}$ for each trial. No lumps were presented in these trials, and $P$ was so informed. $E$ further shaped search topography during these practice trials by physically guiding $P$ 's hand over missed areas as they were missed and instructing more or less pressure and/ or time when appropriate. $P$ was instructed always to use this search pattern during free search trials.

(Manuscript received January 22, 1982; revision accepted for publication June 1,1982 .) 